\title{
The heliospheric modulation of cosmic ray boron and carbon
}

\author{
M. S. Potgieter and U. W. Langner \\ School of Physics, North-West University, 2520 Potchefstroom, South Africa \\ Received: 14 May 2004 - Revised: 30 June 2004 - Accepted: 15 July 2004 - Published: 3 November 2004
}

\begin{abstract}
The observed boron to carbon ratio (B/C) at Earth provides a good measure of the overall secondary to primary ratio of galactic cosmic rays. This makes $\mathrm{B} / \mathrm{C}$ an important constraint and test for the validity and general applicability of theoretical and numerical models of galactic propagation and heliospheric modulation. For this purpose, the modulation
\end{abstract} of boron and carbon in the heliosphere must be understood in greater detail. The latest approach to heliospheric modulation, using a numerical model containing a termination shock, a heliosheath and particle drifts, is used to the study the modulation of the two species. This model also includes a more comprehensive set of diffusion coefficients. From this and previous work follows that the model is compatible with a variety of observations, for seven species, i.e. protons, anti-protons, electrons, positrons, helium, boron, and carbon, with the same set of parameters for both solar magnetic polarity cycles. Despite the rather flat interstellar spectrum for carbon below $100 \mathrm{MeV} / \mathrm{nuc}$, the modulated spectra at $1 \mathrm{AU}$ look very similar for boron and carbon, caused by adiabatic energy losses, implying that the carbon modulation should have a much larger radial gradient in the outer heliosphere below $\sim 200-500 \mathrm{MeV} /$ nuc than boron. Significant modulation can be caused by the heliosheath but it is strongly dependent on energy and on the field polarity, with almost no effect at high energies to the largest effect at low energies. The solar wind termination shock has an important effect on the B to C ratio in the heliosphere, although small at Earth, during the $\mathrm{A}<0$ cycle, with $E<\sim 600 \mathrm{MeV} / \mathrm{nuc}$, but it seems less significant for the $\mathrm{A}>0$ cycle and with increasing tilt angles. Drift models produce different spectra for consecutive solar minimum conditions which may account for the modulation level differences between observations around $100 \mathrm{MeV} /$ nuc compared to around $500 \mathrm{MeV} /$ nuc. All factors taken into account, heliospheric modeling indicates that the interstellar spectra for $\mathrm{B}$ and $\mathrm{C}$ need further refinement around $1 \mathrm{GeV} /$ nuc, in order to fit observations over a wide energy range at Earth and that this refinement probably has to take into account the proposed contribution of a local interstellar carbon component. These results confirm that this numerical model with a TS can reasonably reproduce the $\mathrm{B}$ and $\mathrm{C}$ modulation between the outer boundary and Earth, making it a reasonable

Correspondence to: $\mathrm{M}$. S. Potgieter

(fskmsp@puk.ac.za) approximation for both polarity cycles from solar minimum to moderate solar activity.

Key words. Interplanetary physics (Cosmic rays; Heliopause and solar wind termination shock) - Space plasma physics (Transport processes)

\section{Introduction}

A numerical model describing cosmic ray modulation in the heliosphere, including the solar wind termination shock (TS) and the heliosheath, is applied to the modulation of galactic boron (B) and carbon (C). This model has already been applied to the modulation of cosmic ray protons, antiprotons, electrons, positrons and helium $(\mathrm{He})$ by Langner et al. (2003), Langner and Potgieter (2004a,b), and Potgieter and Langner (2004). This approach makes it possible to determine the modulation of $\mathrm{B}$ and $\mathrm{C}$ in the heliosphere more accurately than before, taking into account the effects of gradient, curvature and neutral sheet drifts, the effects of the TS, and what role the heliosheath and other modulation effects may play, e.g. increasing solar activity by changing the current sheet tilt angle, and to subsequently compute the $\mathrm{B}$ to $\mathrm{C}$ ratio $(\mathrm{B} / \mathrm{C})$ at Earth.

The observed $\mathrm{B} / \mathrm{C}$ provides a good measure of the overall secondary to primary ratio of cosmic rays in the interstellar medium because most of the $\mathrm{B}$ is produced by the spallation of $\mathrm{C}$ when it interacts with interstellar matter during its propagation in the Galaxy, and that $\mathrm{C}$ is only slightly contaminated by secondary $\mathrm{C}$. These properties make the $\mathrm{B} / \mathrm{C}$ an important constraint and a test for the general applicability of theoretical and numerical models of galactic propagation (e.g. Wandel et al., 1987) and heliospheric modulation. For example, Moskalenko et al. $(2002,2003)$ found that by matching the $\mathrm{B} / \mathrm{C}$ using reacceleration models for galactic propagation leads to values of the galactic spatial diffusion coefficients that are too large to produce the required anti-proton flux, when the propagated nucleon spectra are tuned to match the local proton and He flux measurements. They argued that one possibility to avoid this shortcoming could be to reconcile $\mathrm{B} / \mathrm{C}$ with the required flux of secondary anti-protons by including a new, local, "unprocessed" interstellar component at low energies to spectra of primary 
nuclei, thus decreasing the measured secondary/primary ratio. The source of this component, according to Moskalenko et al. (2003), may be associated with the Local Bubble and they argue that independent evidence for supernova activity in the solar vicinity in the last few Myr supports this idea. The observed B/C at Earth and at Voyager 1 and 2 (Webber et al., 2002) is thus important when calculating interstellar spectra (IS) for other species, e.g. anti-protons. It is used as a probe to not only test the numerical heliospheric modulation models, but also the computed IS, and local IS values of galactic propagation models (e.g. Strong et al., 1994, 2000; Webber et al., 2002; Moskalenko et al., 2002, 2003). For this purpose, the modulation of $\mathrm{B}$ and $\mathrm{C}$ in the heliosphere must be understood well. Therefore, we apply the most upto-date approach to heliospheric modulation, using a numerical model containing a solar wind TS and a heliosheath.

The importance of modulation that may occur in the heliosheath has recently been emphasized by cosmic ray observations in the distant heliosphere (e.g. McDonald et al., 2000; Webber et al., 2001). Studying the role of the TS and that of the heliosheath has become most relevant since Voyager 1 is supposedly in the vicinity of the TS (Stone and Cummings, 2003; McDonald et al., 2003) or may have even crossed it (Krimigis et al., 2003). Concerning modulation mechanisms, large-scale gradient, curvature and current sheet drifts that charged particles experience in the global heliospheric magnetic (HMF) are most prominent, if not the dominating mechanism at solar minimum, at least for charged nuclei. Drift models predict, apart from a clear charge-sign dependence (Heber et al., 2003; Ferreira et al., 2003), different spectra for sequential solar minima based on the polarity of the solar magnetic field (Reinecke and Potgieter, 1994). For recent reviews on heliospheric modulation, see Potgieter et al. (2001); Heber (2001); Zhang (2003); and Ferreira and Potgieter (2004a).

The TS can be described as a collisionless shock, that is, a discontinuous transition from supersonic to subsonic flow speed accompanied by discontinuous increases in the number density, temperature and pressure (for an excellent review, see Fichtner, 2001). The region between the heliopause and the TS is known as the heliosheath. There is a reasonable consensus that the TS should be close to 90 AU (e.g. Stone and Cummings, 2001), but the position of the heliopause (assumingly the outer modulation boundary) is less certain, probably at least $30-50 \mathrm{AU}$ beyond the TS in the direction in which the heliosphere is moving (heliospheric nose) but significantly larger in the tail regions. This is because the geometry of the heliosphere should be affected by the relative motion of the heliosphere through the local interstellar medium (e.g. Scherer and Fahr, 2003; Zank and Müller, 2003). These effects are not incorporated in the present two-dimensional TS modulation model.

Modeling the heliospheric modulation of galactic B and $\mathrm{C}$ has not been addressed recently, except with a force-field approach to modulation (Webber et al., 2002; Moskalenko et al., 2002, 2003). A force-field approach is a reasonable approximation for the modulation galactic cosmic rays at Earth but its accuracy decreases rapidly with increasing radial distance (see also Caballero-Lopez and Moraal, 2004) and towards the heliospheric poles. Revisiting the modulation of $\mathrm{B}$ and $\mathrm{C}$ has become appropriate because of improved IS computations and a new approach to heliospheric diffusion coefficients, using a TS modulation model, including a heliosheath and drifts. This will further test and illustrate the general applicability of the TS modulation model and the set of heliospheric diffusion parameters for the various cosmic ray species for both polarity cycles. In combination this has not been done before for B and C. The two sets of IS for B and $\mathrm{C}$ used in this study were computed by Moskalenko et al. $(2002 ; 2003)$ with a comprehensive galactic propagation model.

The following topics are addressed in particular: 1) The modulation of galactic B and C in a simulated heliosphere for both HMF polarity cycles as solar modulation changes from minimum to moderate maximum conditions. 2) The basic differences in the modulation of $\mathrm{B}$ and $\mathrm{C}$ when using a modulation model with a TS and then without a TS. 3) The level and the importance of modulation in the heliosheath for both species, and 4) to establish the consequent modulation effects on the modulated B/C at Earth using two sets of IS based on different assumptions for galactic propagation.

\section{Modulation model}

The model is based on the numerical solution of the timedependent cosmic ray transport equation (Parker, 1965):

$$
\begin{aligned}
\frac{\partial f}{\partial t} & =-\left(\mathbf{V}+\left\langle\mathbf{v}_{D}\right\rangle\right) \cdot \nabla f+\nabla \cdot\left(\mathbf{K}_{s} \cdot \nabla f\right) \\
& +\frac{1}{3}(\nabla \cdot \mathbf{V}) \frac{\partial f}{\partial \ln p}+J_{\text {source }},
\end{aligned}
$$

where $f(\mathbf{r}, p, t)$ is the omnidirectional cosmic ray distribution function, $p$ is the particle momentum, $\mathbf{r}$ is position, and $t$ is time, with $\mathbf{V}$ the solar wind velocity. The terms on the right-hand side represent convection, gradient and curvature drifts, diffusion, adiabatic energy changes and a source function, respectively. The tensor $\mathbf{K}_{S}$ consists of a parallel diffusion coefficient $\left(\kappa_{\|}\right)$, and perpendicular diffusion coefficients $\left(\kappa_{\perp}\right)$. The averaged guiding centre drift velocity for a near isotropic cosmic ray distribution is given by $\left\langle\mathbf{v}_{D}\right\rangle=\nabla \times\left(\kappa_{T} \mathbf{e}_{B}\right)$, with $\mathbf{e}_{B}=\mathbf{B} / B_{m}$, where $B_{m}$ is the magnitude of the modified background HMF as described below, and with $\kappa_{T}$ (or sometimes indicated as $\kappa_{A}$ ) the diffusion coefficient specified by the off-diagonal elements of the generalized diffusion tensor $\mathbf{K}$, which describes gradient and curvature drifts in the large-scale HMF. The function $J_{\text {source }}$ represents any local source, e.g. the Jovian electrons, the pickup ions, etc., which is neglected for this work. The transport equation is solved time-dependently in a spherical coordinate system as a combined diffusive shock acceleration and drift modulation model with two spatial dimensions, neglecting any azimuthal dependence and is symmetric around the equatorial plane. A smilar two-dimensional shock acceleration numerical model was described orginally by Jokipii 
et al. (1993); see also e.g. Steenkamp (1995), Haasbroek et al. (1997) and Potgieter and Ferreira (2002). The HMF was assumed to have a basic Parkerian geometry in the equatorial plane but was modified in the polar regions similar to the approach of Jokipii and Kóta (1989).

The outer modulation boundary was assumed at $r_{b}=120 \mathrm{AU}$, where the interstellar spectra of Moskalenko et al. $(2002 ; 2003)$ for $\mathrm{B}$ and $\mathrm{C}$ are specified. The TS is assumed at $r_{s}=90 \mathrm{AU}$ with a compression ratio $s=3.2$ and a shock precursor scale length of $L=1.2 \mathrm{AU}$ (le Roux et al., 1996; Langner et al., 2003). This means that up to the shock, the solar wind speed $V$ decreases by $0.5 s$ starting at $L$, then abruptly as a step function to the downstream value, in total to $V / s$. The HMF thus increases by a factor $s$ at the TS. Beyond the TS, $V$ assumingly decreases further as $1 / r^{2}$ to the outer boundary, which implies that no additional acceleration can occur beyond the shock and that adiabatic energy losses become insignificant, which may be an oversimplification. Because of the assumption of axial-symmetry, the model is applicable only in the direction of the heliospheric nose (where the Voyager spacecraft are moving) and in the direction of the heliospheric tail.

Concerning the solar wind, it is assumed that $V$ changes from $400 \mathrm{~km} / \mathrm{s}$ in the equatorial plane $\left(\theta=90^{\circ}\right)$ to $800 \mathrm{~km} / \mathrm{s}$ in the polar regions. This factor of a 2.0 increase happens in the heliosphere for $60^{\circ} \geq \theta \geq 300^{\circ}$ and for $240^{\circ} \geq \theta \geq 120^{\circ}$ for solar minimum conditions, but it is reduced to a factor of 1.10 with $10^{\circ} \geq \theta \geq 350^{\circ}$ and for $190^{\circ} \geq \theta \geq 170^{\circ}$ for moderate maximum conditions. A modified version of the current sheet model of Hattingh and Burger (1995), which emulates the waviness of the current sheet in a two-dimensional heliosphere, was used (see also Langner, 2004). The current sheet tilt angles, as calculated by Hoeksema (Wilcox Solar Observatory; courtesy of J. T. Hoeksema: http://wso.stanford.edu), were assumed to represent solar minimum and moderate maximum modulation conditions with $\alpha=10^{\circ}$ and $\alpha=75^{\circ}$, respectively, during $\mathrm{A}>0$ (e.g. $\sim 1990-2001$ ) and $\mathrm{A}<0$ (e.g. $\sim 1980-1990$ ) magnetic polarity cycles. This TS model does not describe extreme solar maximum conditions; a different code has to be used for that purpose (see Ferreira and Potgieter, 2004b).

The diffusion coefficients $\kappa_{\|}, \kappa_{\perp}$, and $\kappa_{T}$ are based on those given by Burger et al. (2000) for a steady-state model, except for changes caused by the introduction of the TS in this model (Langner et al., 2003). Perpendicular diffusion is assumed to enhance towards the poles in order to fit the observed latitudinal gradients (e.g. Burger et al., 2000). For a complete description of these diffusion coefficients, see Langner et al. (2003). They are optimal for a numerical TS model without an azimuthal dependence and without solar maximum transient effects, e.g. global merged interaction regions. This set can also be used by changing only the rigidity dependences of $\kappa_{\|}$accordingly at low rigidities for electrons and positrons (Potgieter and Langner, 2004) to give reasonable fits to a variety of data sets and is the same for both polarity cycles. The details of the model were described by Langner et al. (2003) and Langner and Potgieter (2004a).

\section{Results and discussion}

The modulation obtained with the TS model with respect to the IS for B and C (Moskalenko et al., 2002) as a function of kinetic energy is shown in the left panels of Figs. 1 and 2, respectively. This is done at 1,60 , and $90 \mathrm{AU}$ in the equatorial plane for the $\mathrm{A}>0$ and $\mathrm{A}<0$ polarity cycles with $\alpha=10^{\circ}$ and $\alpha=75^{\circ}$, respectively. The right panels of Figs. 1 and 2 show the corresponding differential intensities at 0.016, 0.2 and $1.0 \mathrm{GeV} /$ nuc as a function of radial distance in the equatorial plane. In Fig. 1 the solutions are shown, respectively, for a model with a TS and then without a TS in the model. Comparing the energy spectra and radial dependence of the intensities for the chosen energies in these two figures, the following are noted: 1) In general, the modulation for B and $\mathrm{C}$ differs as a function of radial distance primarily because of the much steeper spectral slope for the IS below $100 \mathrm{MeV} /$ nuc for B compared to C. This implies that if the IS were correct, the $\mathrm{C}$ modulation should have a much larger radial gradient below $\sim 200-500 \mathrm{MeV} /$ nuc in the outer heliosphere than for B. 2) The spectra differ significantly when the HMF switches polarity from $A>0$ to $A<0.3$ ) The spectral slopes at low energies change with increasing radial distance as the adiabatic energy loss effect becomes less; it is also dependent on the HMF polarity. Despite the rather flat IS for $\mathrm{C}$ below $100 \mathrm{MeV} / \mathrm{nuc}$, the modulated spectra at $1 \mathrm{AU}$ look very similar for $\mathrm{B}$ and $\mathrm{C}$, a characteristic of large adiabatic "cooling". 4) The modulation of B and C is affected by incorporating a TS, evident from comparing solutions with and without a TS in the model. 5) A "barrier" type modulation is caused by the heliosheath. It differs significantly for different energies, from almost no effect at high energies to the largest effect at low energies, and with the change in HMF polarity. 6) Also note the manner in which the modulation changes from solar minimum to moderate solar maximum activity.

From Figs. 1 and 2 follow that the modulated B and C spectra at large radial distances $\left(r \rightarrow r_{s}\right)$ for the $\mathrm{A}<0$ cycle exceed the corresponding IS between $\sim 200 \mathrm{MeV} /$ nuc and a few $\mathrm{GeV} /$ nuc, owing to the presence of the TS, as has been noted for cosmic ray protons and He (Langner et al., 2003; Langner and Potgieter, 2004b). The effect of the TS on the modulation of $\mathrm{B}$ and $\mathrm{C}$ with respect to the IS is for the larger part of the heliosphere significant; it drastically decreases the intensities at lower energies (e.g. at $100 \mathrm{MeV} /$ nuc) but increases it at higher energies (e.g. at $1 \mathrm{GeV} / \mathrm{nuc}$ ), as the lower energy particles are being accelerated to higher energies. The adiabatic spectral slopes are also altered in the process. This effect seems not as pronounced for larger tilt angles (increased solar activity) and clearly depends on the particle drift direction.

The modulation in the heliosheath (in this case, for the upstream and down-stream regions, with respect to the direction in which the heliosphere is moving) is clearly an important part of the total modulation for $\mathrm{B}$ and $\mathrm{C}$, as shown in the right panels of Figs. 1 and 2. The TS plays in this regard a prominent role and can be regarded as a main contributor to 

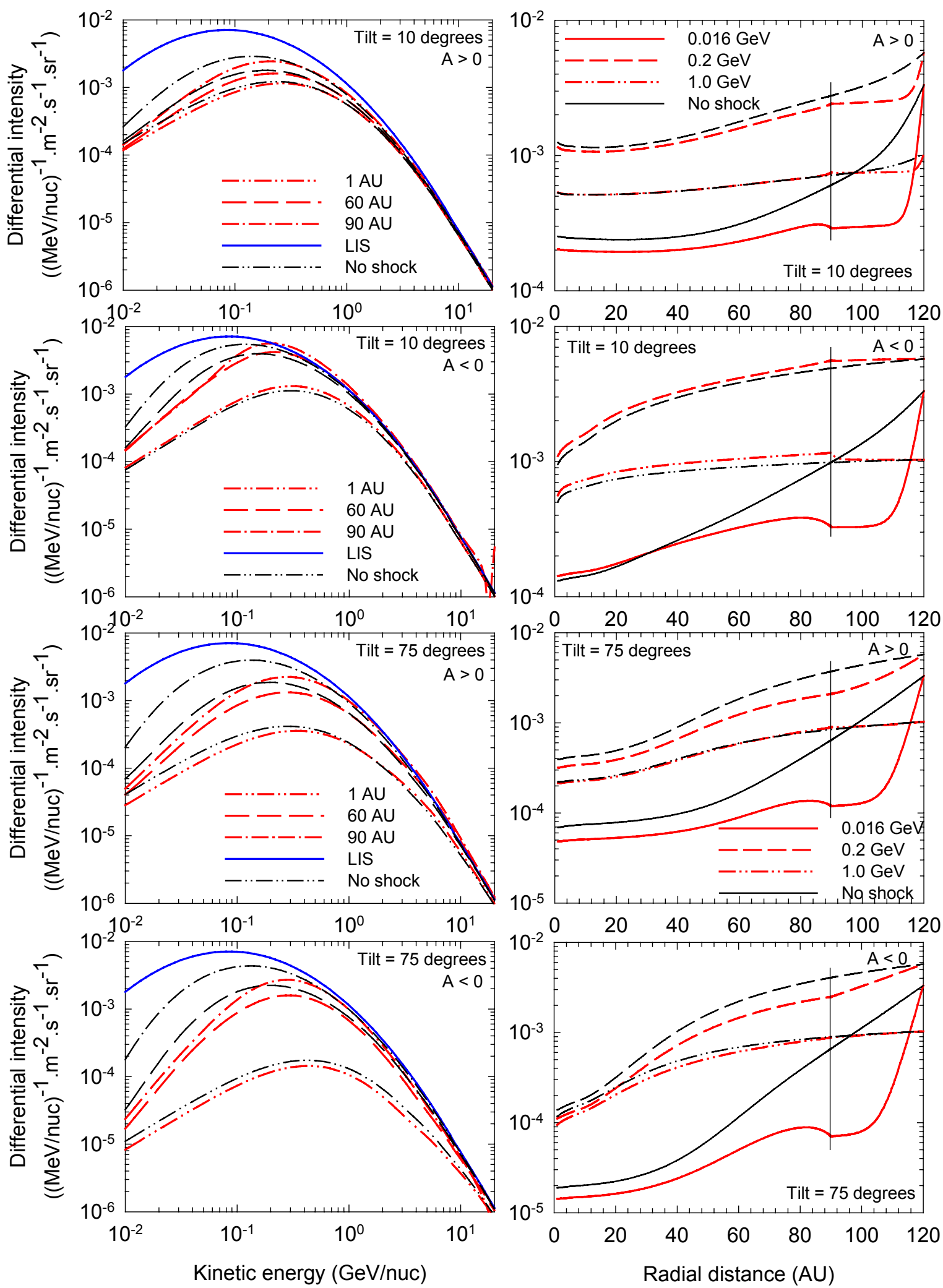

Fig. 1. Left panels: Computed differential intensities for galactic boron (B) as a function of kinetic energy for both polarity cycles, at radial distances of 1, 60, $90 \mathrm{AU}$ (bottom to top) in the equatorial plane. Right panels: The corresponding differential intensities as function of radial distance for $0.016,0.2$ and $1.0 \mathrm{GeV}$, respectively. In all panels the TS is at $90 \mathrm{AU}$, as indicated, with the IS (blue lines) specified at $120 \mathrm{AU}$, with $\alpha=10^{\circ}$ and $75^{\circ}$, respectively. Solutions without a TS in the model are given as black lines for the same radial distances and energies. Note the scale difference between some of the panels. 

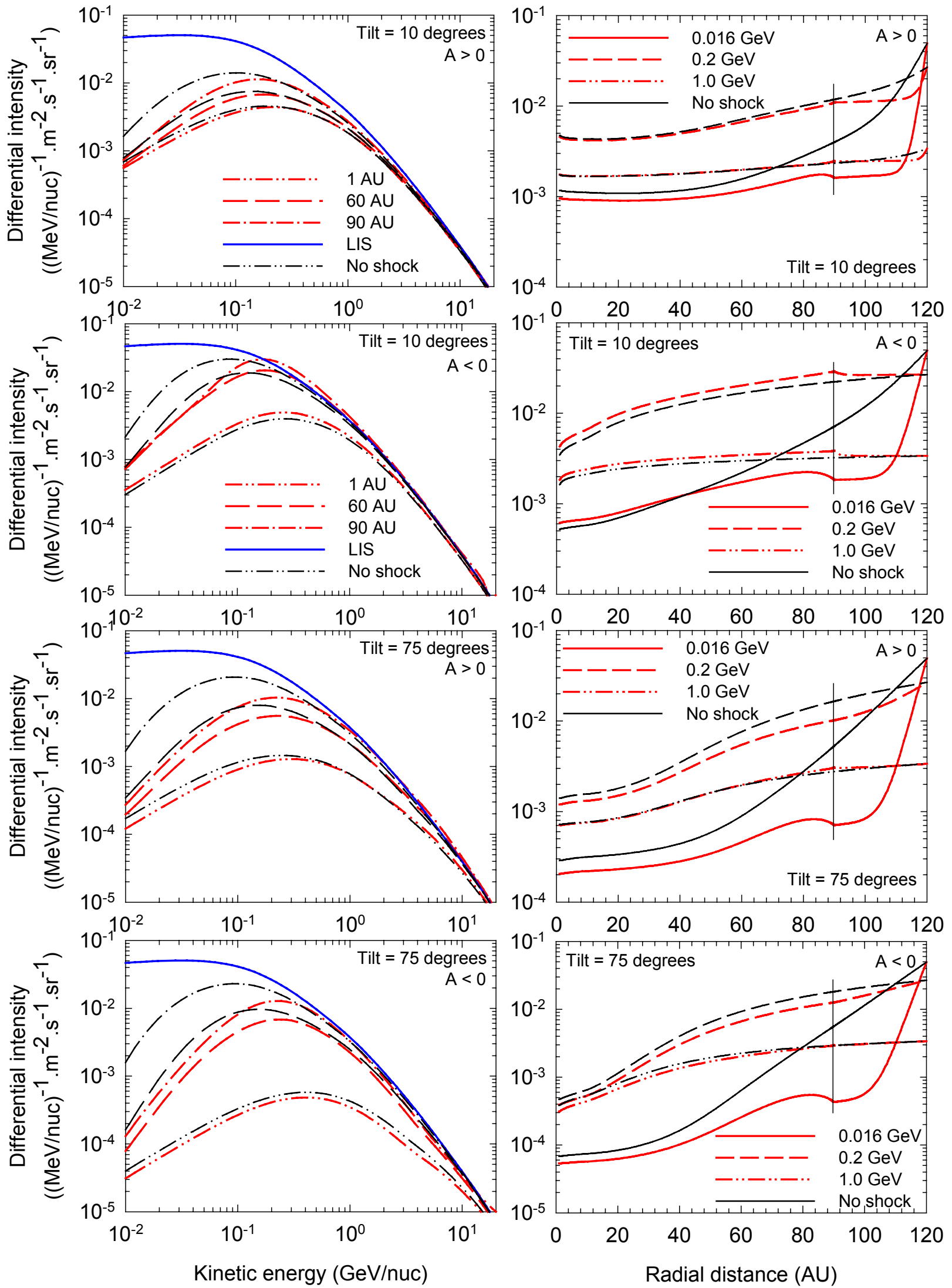

Fig. 2. Similar to Fig. 1 but for carbon (C). 
the so-called "barrier" modulation effect at low energies. For both species its effect becomes clearly more pronounced the lower the energy, as has also been seen for cosmic ray protons and He. At higher energies, the effect of the heliosheath progressively diminishes; the radial dependence beyond the shock may vanish or even becomes negative, to create a conspicuous shock effect on the radial intensity profiles. This effect is strongly dependent on the HMF polarity cycles. At these energies spacecraft would measure more or less constant intensities beyond the shock and almost up to the heliopause and for both polarity cycles at solar minimum activity. For an elaborate discussion on these effects for protons, see also Langner et al. (2003). It is expected that in the downstream direction, towards the tail of the heliosphere, the heliosheath is considerably wider and consequently may have an extended "barrier" type effect on cosmic ray modulation, differing quantitatively but not qualitatively from what has been presented here.

In Fig. 3 the effects of the TS on B and C modulation are emphasized by depicting the ratio of intensities obtained with and without a TS in the model as a function of kinetic energy at radial distances of 1,60 , and $90 \mathrm{AU}$, and as a function of radial distance at energies of $0.016,0.2$ and $1.0 \mathrm{GeV} / \mathrm{nuc}$, respectively, in the equatorial plane for both polarity cycles when $\alpha=10^{\circ}$. The modulation parameters of the two approaches are kept the same for these calculations, in order to quantify the effects of the TS on the modulation of these species. The deviation from unity is an indication of the role of the TS. Note that the ratios as a function of energy converge naturally at $E>\sim 10 \mathrm{GeV} /$ nuc because the TS has progressively less modulation effects the higher the energy becomes, and that the ratios as a function of radial distance approach unity at $120 \mathrm{AU}$, where the IS are specified. Evidently, the effect of the TS on the modulation of galactic B and $\mathrm{C}$ with respect to the relevant IS is significant at lower energies but it becomes less the deeper one penetrates into the heliosphere, as expected. The differences between the two approaches are most significant with $E<\sim 100 \mathrm{MeV} /$ nuc and $r>\sim 60 \mathrm{AU}$, similar to cosmic ray protons and $\mathrm{He}$. The ratios have the lowest values at $\sim 115 \mathrm{AU}$ for $\mathrm{A}>0$ at all energies, which indicates that the effect of the TS model is prominent at these larger distances. This minimum value occurs at smaller radial distances for the $\mathrm{A}<0$ polarity cycle. The ratios in the left panels also have a minimum at a certain energy which becomes smaller and moves to lower energies as the radial distance increases.

The inclusion of the TS in the model can influence the modulation of B and C, even at Earth, although little in the inner heliosphere. As shown in Figs. 1 and 2, the B and C intensities at low energies are lower at Earth with the TS than without it in the $\mathrm{A}>0$ polarity cycle, but not for the $\mathrm{A}<0$ cycle, emphasizing the role of particle drifts. In Fig. 4 this effect is further illustrated when $B / C$, as obtained with a TS and then without a TS in the model, is shown as a function of kinetic energy at Earth for both polarity cycles when $\alpha=10^{\circ}$ and $\alpha=75^{\circ}$, respectively. Evidently, there is an effect on the ratio at Earth during the $\mathrm{A}<0$ cycle with $E<\sim 600 \mathrm{MeV} /$ nuc but it seems insignificant for the $\mathrm{A}>0$ cycle, and with increasing tilt angles.

Next, the role of the heliosheath is further illustrated and discussed. The computed modulation to take place in the heliosheath, between $r_{b}$ and $r_{s}$, is compared to what happens between $r_{b}$ and $1 \mathrm{AU}$ (IS to Earth) and between $r_{s}$ and $1 \mathrm{AU}$ (TS to Earth). This comparison is emphasized by showing in Fig. 5 the intensity ratios $j_{\text {LIS }} / j_{1}, j_{\text {LIS }} / j_{90}$ and $j_{90} / j_{1}$ for $\mathrm{B}$ and $\mathrm{C}$ as a function of kinetic energy in the equatorial plane for both polarity cycles with $\alpha=10^{\circ}$. Note that for a few cases the ratios become less than unity. Obviously, all these ratios must converge at a high enough energy where no modulation takes place. According to this figure a significant level of modulation occurs in the heliosheath when $\mathrm{A}>0$ with $E<\sim 200 \mathrm{MeV} /$ nuc for solar minimum $\left(\alpha=10^{\circ}\right)$. This is also true for $\mathrm{A}<0$ but at a somewhat lower energy. The level of modulation in the heliosheath decreases significantly for $E>200 \mathrm{MeV} /$ nuc in contrast with that of $j_{90} / j_{1}$ for the $\mathrm{A}<0$ cycle but to a lesser extent for the $\mathrm{A}>0$ cycle.

In Fig. 6 the computed differential intensities for B and $\mathrm{C}$ are shown at Earth for both polarity cycles compared to B observations from the HEAO-3 experiment (Engelmann et al., 1990) and C observations (e.g. Stephens and Streitmatter, 1998; the detailed data compilation was done by Moskalenko et al., 2003). These comparisons are shown for two sets of IS, the first set calculated by Moskalenko et al. (2002) and the second set calculated by Moskalenko et al. (2003), using their galactic propagation model. This second approach contains a new, local component to spectra of primary nuclei and is probably closer to what can be considered a local IS. The $\mathrm{B}$ to $\mathrm{C}$ ratios at $1 \mathrm{AU}$ and $60 \mathrm{AU}$, as a function of kinetic energy are also shown for both polarity cycles with $\alpha=10^{\circ}$, compared to the observations, with the interstellar $\mathrm{B} / \mathrm{C}$ (at $120 \mathrm{AU}$ ) as a reference. The difference between the two IS for $\mathrm{B}$ and for $\mathrm{C}$ is the same and consequently has an insignificant effect on $\mathrm{B} / \mathrm{C}$ (which illustrates that if one fits the $\mathrm{B} / \mathrm{C}$ ratio at Earth, one does not necessarily fit the modulated spectra). The computations are done with the TS included in the model.

First, the model solutions are discussed. As noted before the spectral shapes at $1 \mathrm{AU}$ are very similar for $\mathrm{B}$ and $\mathrm{C}$, owing to adiabatic energy loses between $120 \mathrm{AU}$ and 1 AU. This causes a steady B/C below 200-300 MeV/nuc. This ratio will systematically decrease with increasing radial distances to eventually coincide with the IS ratios. However, the spectral slopes at $1 \mathrm{AU}$ are slightly different for the two polarity epochs, owing to the different particle drift directions during the two magnetic polarity cycles. This causes the well-known crossing of the spectra for successive solar minima, seen here between 100-200 MeV/nuc (see also Reinecke and Potgieter, 1994). Concerning the compatibility between the observations and the model solutions for the IS of Moskalenko et al. (2002), it is most reasonable above $500 \mathrm{MeV} / \mathrm{nuc}$, but the excellent fit to $\mathrm{B} / \mathrm{C}$ below $300 \mathrm{MeV} /$ nuc is fortuitous because for both $\mathrm{C}$ and $\mathrm{B}$ the observations are higher by the same margin than the modeling result at these energies. However, a more reasonable fit 

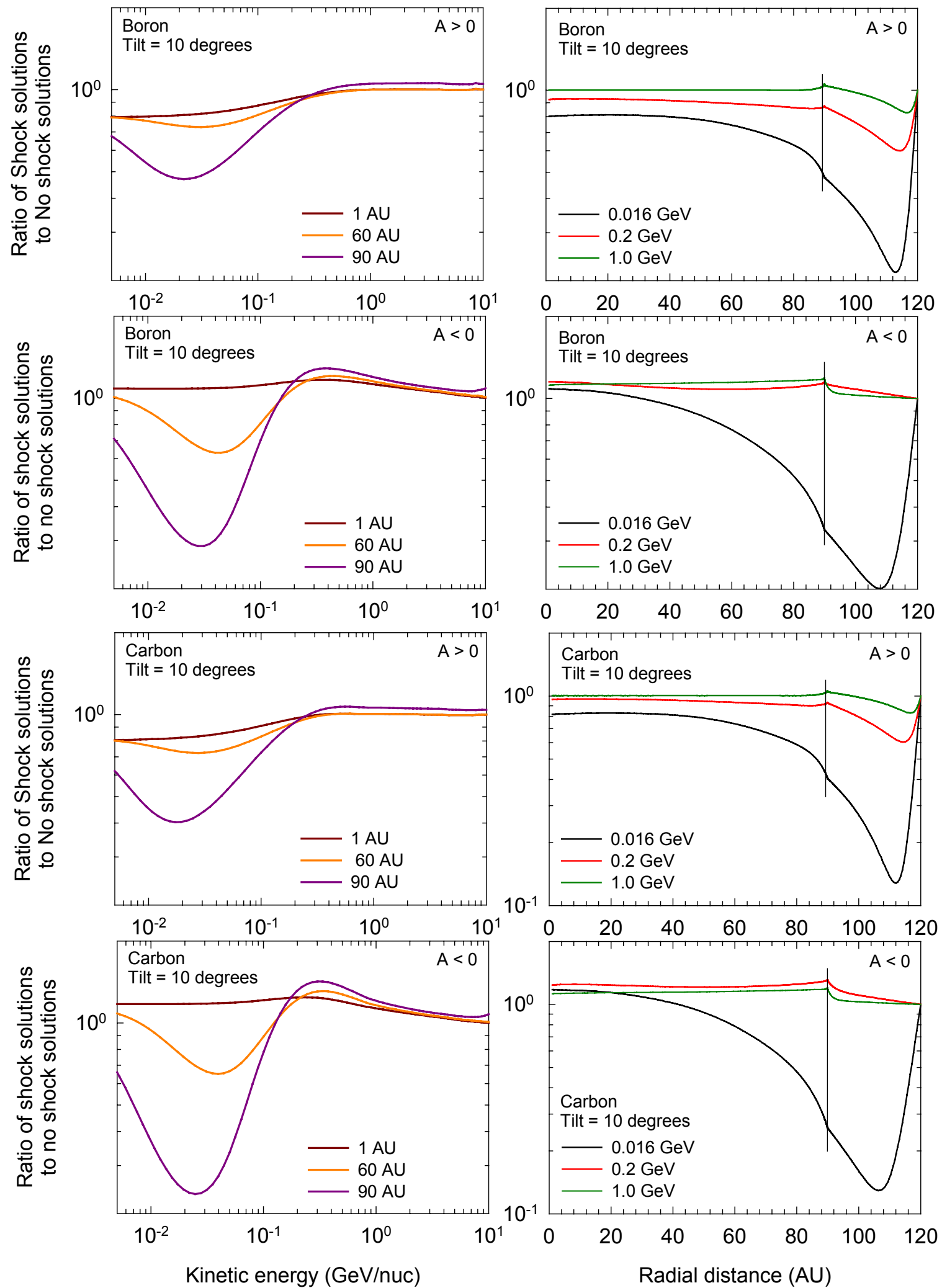

Fig. 3. Intensity ratios of solutions for B (top) and C (bottom) with a TS model compared to those without a TS in the model as a function of kinetic energy at radial distances of 1,60 , and $90 \mathrm{AU}$ (left panels) and as a function of radial distance at energies of $0.016,0.2$ and $1.0 \mathrm{GeV}$ (right panels) for both polarity cycles in the equatorial plane, with $\alpha=10^{\circ}$. 

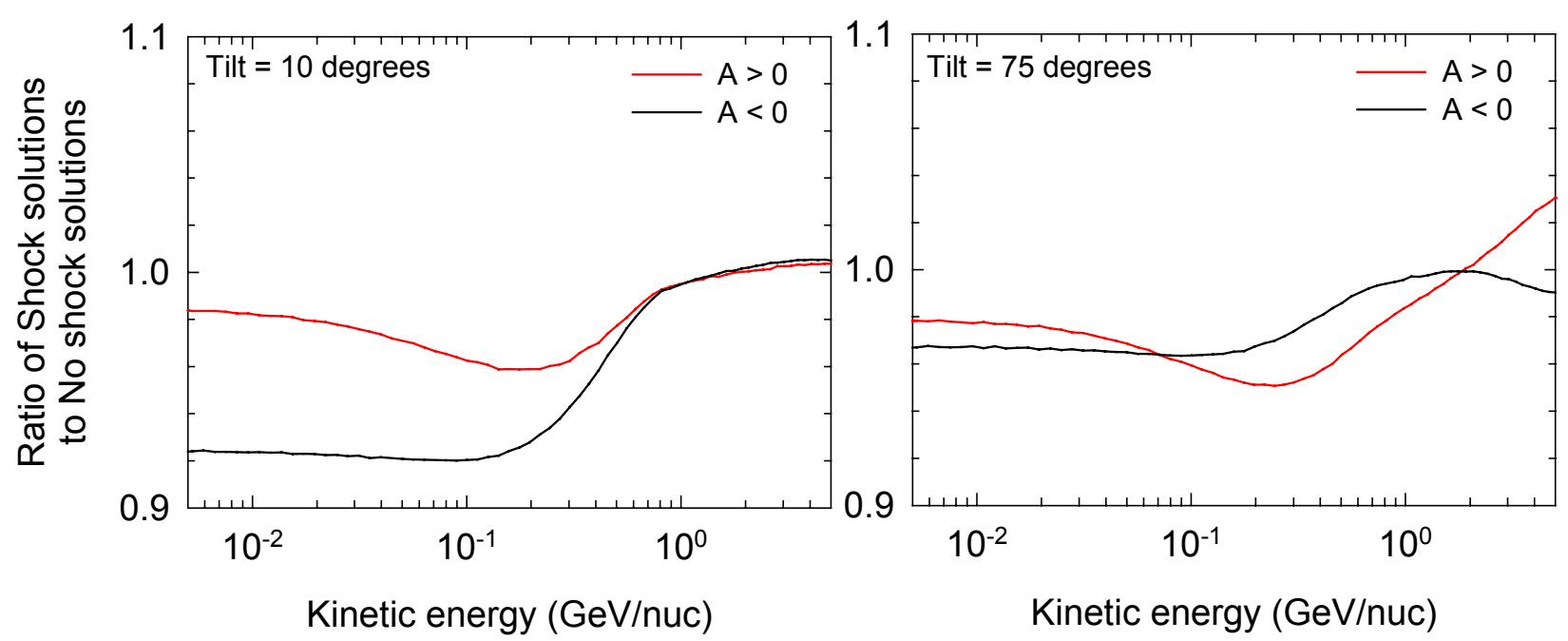

Fig. 4. Computed intensity ratios, B/C, with a TS in the model compared to those without a TS as a function of kinetic energy at Earth for both polarity cycles with $\alpha=10^{\circ}$ and $75^{\circ}$, respectively.
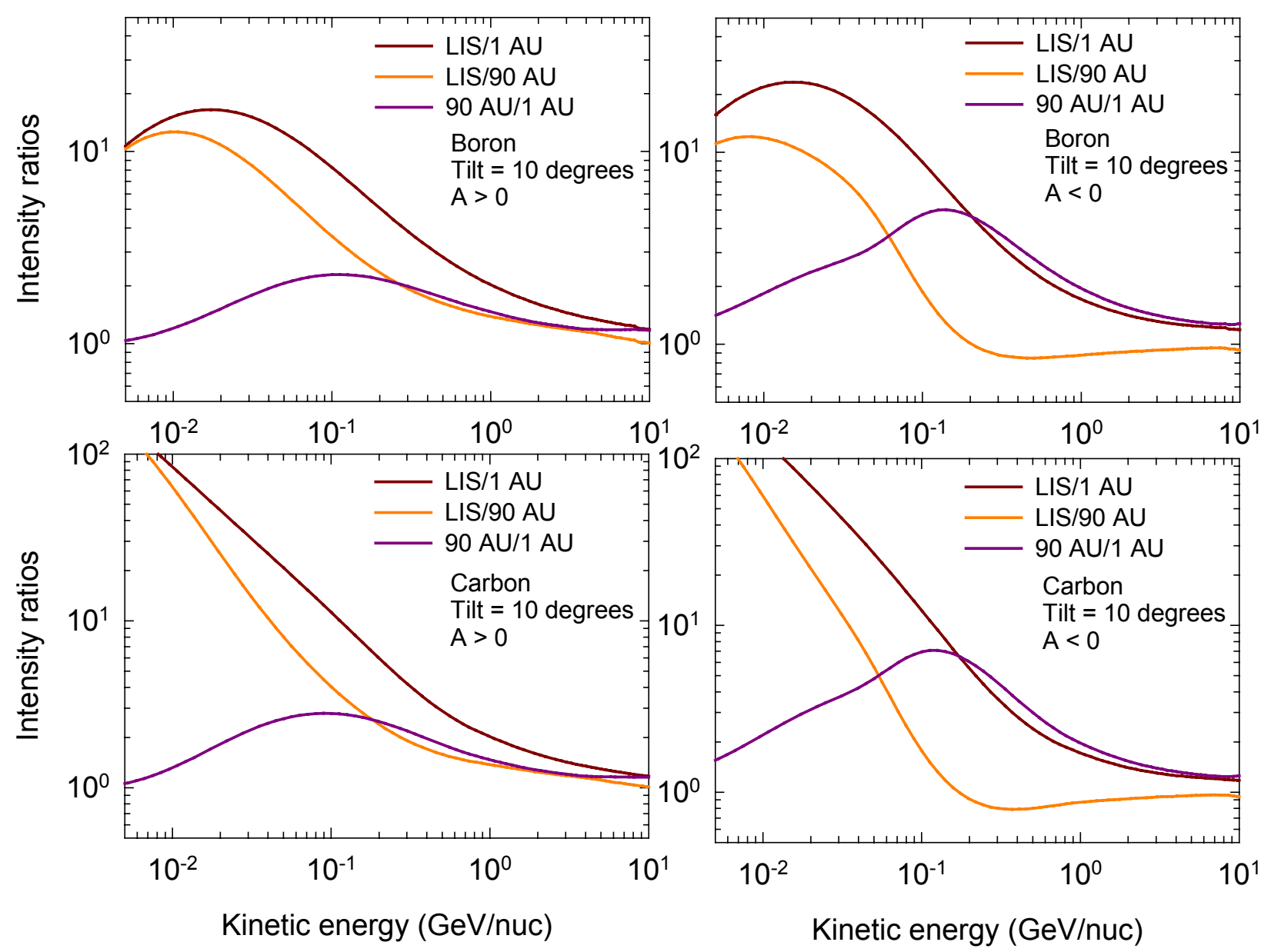

Fig. 5. Intensity ratios $j_{\mathrm{LIS}} / j_{1}, j_{\mathrm{LIS}} / j_{90}$ and $j_{90} / j_{1}(120$ to $1 \mathrm{AU}, 120$ to $90 \mathrm{AU}$ and 90 to $1 \mathrm{AU})$ for $\mathrm{B}$ and $\mathrm{C}$ as a function of kinetic energy in the equatorial plane with $\alpha=10^{\circ}$; left panels: for $A>0$, right panels for $A<0$. Interstellar spectra are considered local interstellar spectra (LIS) at $120 \mathrm{AU}$ and the TS is positioned at $90 \mathrm{AU}$. Note the scale difference between some of the panels. 

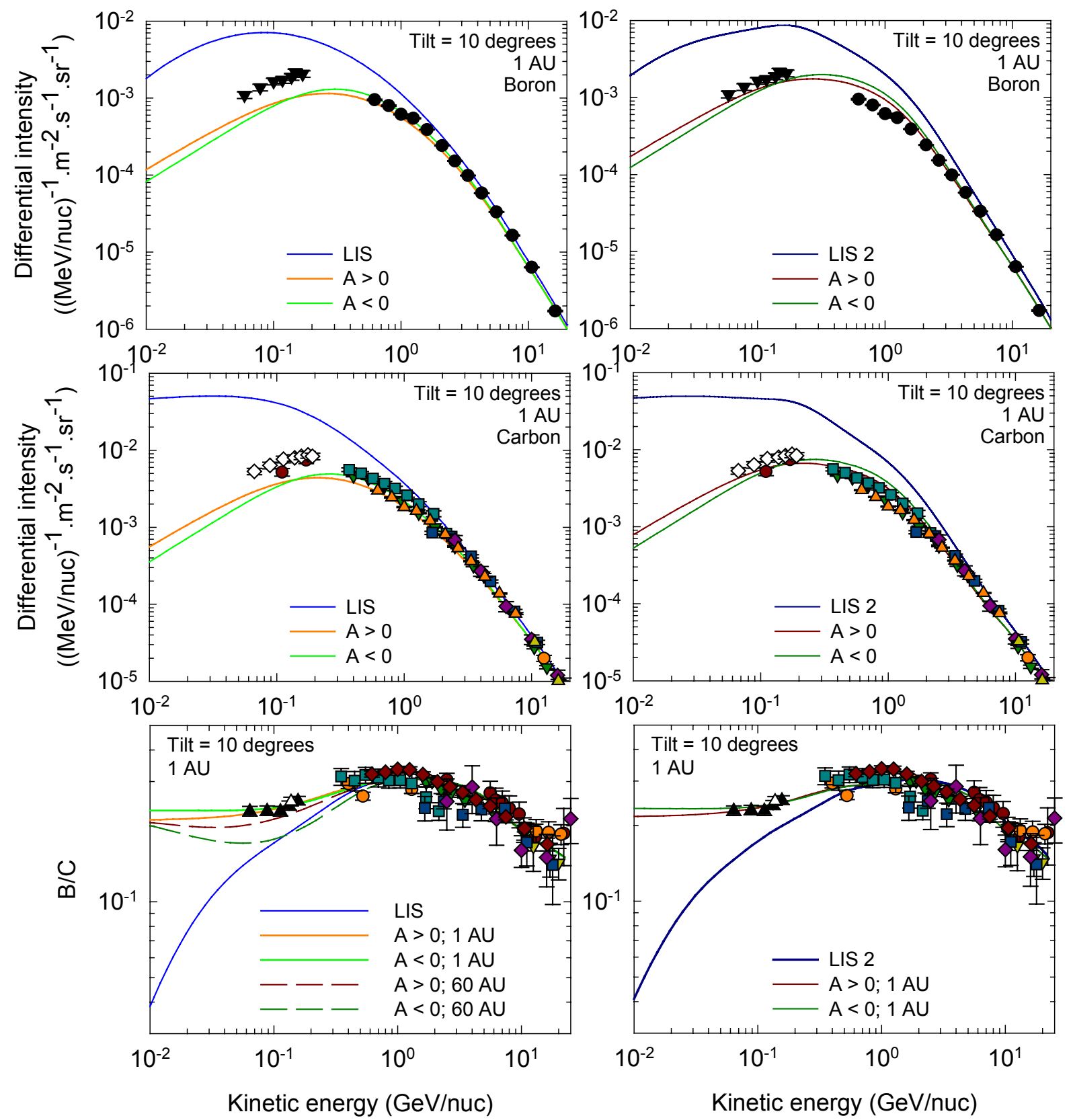

Fig. 6. Top and middle panels: Computed differential intensities for B (top) and C (middle) at Earth for both polarity cycles compared to B observations from the HEAO-3 experiment (Engelmann et al., 1990) and ACE (Davis et al., 2000) and C observations (see Stephens and Streitmatter, 1998 for references), respectively. Computations are done with the IS for B and C by Moskalenko et al. (2002) (left panels) and by Moskalenko et al. (2003) (right panels). Bottom panel: B/C as a function of kinetic energy for both polarity cycles with $\alpha=10^{\circ}$ compared to observations. The computations are done with a TS in the model and are compared to the interstellar B/C (at 120 AU) as a reference (blue lines). The data compilation is taken from Moskalenko et al. (2003).

is obtained in this energy range by using the second set of IS of Moskalenko et al. (2003) which from $\sim 200 \mathrm{MeV} / \mathrm{nuc}$ to $\sim 4 \mathrm{GeV} /$ nuc is higher than the previous one. Unfortunately, these modified IS produce modulated spectra that do not represent the observations well between $\sim 200 \mathrm{MeV} /$ nuc and $\sim 1 \mathrm{GeV} /$ nuc for both $\mathrm{B}$ and $\mathrm{C}$, with the fit to the lowenergy $\mathrm{B} / \mathrm{C}$ still in place. This aspect is emphasized in Fig. 7 by showing the two sets of IS, with the changes introduced by Moskalenko et al. (2003), and the corresponding ratios as a function of energy. The modeling and observation comparison indicates that their new IS seems somewhat too high above $\sim 1 \mathrm{GeV} /$ nuc but still too low below $\sim 1 \mathrm{GeV} /$ nuc, implying that the peak in the ratio between $1-2 \mathrm{GeV} /$ nuc should rather occur around $400 \mathrm{MeV} /$ nuc. 


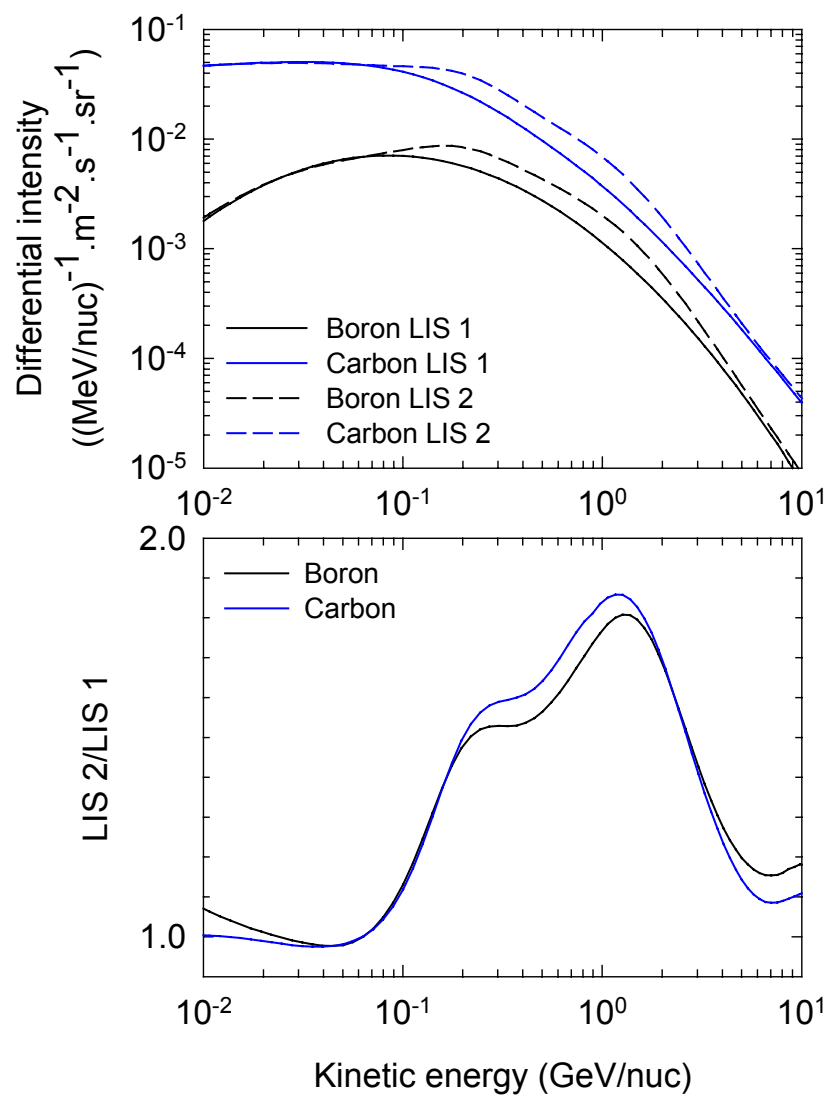

Fig. 7. A comparison of the two sets of interstellar spectra for $\mathrm{B}$ and $\mathrm{C}$ as used in this study; lower values (LIS1; solid lines) by Moskalenko et al. (2002), higher values (LIS2-dashed lines) by Moskalenko et al. (2003). The latter contains a local interstellar contribution to spectra of primary nuclei as proposed by Moskalenko et al. (2003) and is probably closer to what can be considered a local IS for carbon. In the lower panel the corresponding ratios (LIS2/LIS1) are shown as a function of energy/nuc.

The question arises as to what may be responsible for the incompatibility between the otherwise successful modulation model and these observations. There are two main considerations: 1) From a heliospheric point of view, the solar minimum low-energy B and $\mathrm{C}$ observations between 50$200 \mathrm{MeV} /$ nuc are relatively too high with respect to those above $500-600 \mathrm{MeV} / \mathrm{nuc}$; this is the basic problem that we face. Moskalenko et al. (2003) also showed the same "break in modulation level continuity" between the observations for the mentioned energy ranges when using a force-field approach to modulation (see their Fig. 10). However, for the purpose of this paper we assume these $B$ and $C$ observations to be reliable. It is then not possible to fit both energy ranges with a modulation model as used for this work unless the heliospheric diffusion coefficients are "forced" to have a very peculiar heliospheric rigidity dependence that would "bend" the computed modulated B and C spectra from the observed values around $500 \mathrm{MeV} /$ nuc upwards to those below $200 \mathrm{MeV} /$ nuc. Such a change will certainly not fit the low-energy protons and $\mathrm{He}$, so that it is unlikely that the rigidity dependence of the relevant heliospheric diffusion coefficients for nuclei needs such a drastic modification. Another aspect to note is that particle drifts produce clearly different spectra for consecutive solar minimum conditions, with the cross-over of the spectra for $\mathrm{A}<0$ and $\mathrm{A}>0$ occurring around 100-200 MeV/nuc. This value depends on the level of drifts and may be too low in this case, if at higher energies the difference between the solar minima spectra would increase at energies below $\sim 1 \mathrm{GeV} /$ nuc, which might account for the apparent "modulation discontinuity" in the observations between the two mentioned energy ranges. 2) The IS for B and $\mathrm{C}$ need further refinement below a few $\mathrm{GeV} / \mathrm{nuc}$ (to make them true local IS) probably in the sense that either the galactic diffusion coefficients or injection spectra need modification or that the contribution of a local interstellar component, proposed by Moskalenko et al. (2003), is indeed present but not yet refined enough. To explain the B and $\mathrm{C}$ observations at Earth, this proposed additional component should cause the local IS to bend significantly to higher values below $\sim 1 \mathrm{GeV} /$ nuc. Voyager data may assist in determining to what low energy value this component may contribute. At Earth this kind of information is unfortunately "wiped out" by the adiabatic energy losses.

\section{Summary and conclusions}

The heliospheric modulation of galactic $\mathrm{B}$ and $\mathrm{C}$ is described with a numerical model that includes particle drifts, the solar wind termination shock, and a heliosheath beyond the shock. This combined approach has not been done before for B and $\mathrm{C}$, so that with improved IS computations (Moskalenko et al., 2003) and a new approach to heliospheric diffusion coefficients (Burger et al., 2000), studying the modulation of these cosmic ray species has become appropriate. Previously, this model has been applied successfully to the modulation of cosmic ray protons, anti-protons, electrons, positrons and He by Langner et al. (2003), Langner and Potgieter (2004a, 2004b), and Potgieter and Langner (2004). The modeling was focused on several aspects of the modulation of B and C: 1) The basic differences in the heliospheric modulation of galactic B and C. 2) How the inclusion of a TS and a heliosheath in the model alters the modulation of $\mathrm{B}$ and $\mathrm{C}$ and the subsequent effects on the B/C ratio at Earth. 3) The nature of modulation effects to be expected near the TS and in the heliosheath. 4) The effects of increased solar activity and the tilt angle dependence.

Qualitatively, the modulation for B and C is similar to those of protons and He, e.g. despite the rather flat IS for $\mathrm{C}$ below $100 \mathrm{MeV} / \mathrm{nuc}$, the modulated spectra at $1 \mathrm{AU}$ look very similar for $\mathrm{B}$ and $\mathrm{C}$, owing to large adiabatic energy losses. The spectral slopes of the computed spectra at $1 \mathrm{AU}$ are slightly different for the two polarity epochs, owing to the different particle drift directions. This causes the wellknown crossing of the spectra for successive solar minima. The modulation for $\mathrm{B}$ and $\mathrm{C}$ that has been produced with a TS and then without a TS in the model differs significantly, 
depending on the HMF polarity. These differences increase towards lower energies and larger radial distances. The ratio of B to C obtained with a TS in the model and then without a TS shows that termination shock effects may even be expected at Earth.

The heliosheath can be considered a distinguishable modulation "barrier" for B and C, with the overall effect clearly energy, polarity cycle and solar activity dependent, e.g. most of the modulation may occur in the heliosheath for $E<\sim 200 \mathrm{MeV} / \mathrm{nuc}$ at solar minimum during $\mathrm{A}>0$ cycles. These results indicate that the local IS for B and C may not be known at $E<\sim 200 \mathrm{MeV} /$ nuc until a spacecraft actually approaches the heliopause, because of the strong modulation that occurs in the heliosheath. This implies that if the IS were correct, the $\mathrm{C}$ modulation should have a much larger radial gradient below $\sim 200-500 \mathrm{MeV} /$ nuc in the outer heliosphere than B.

The compatibility between the observations at Earth and the model solutions is most reasonable above $\sim 500 \mathrm{MeV} / \mathrm{nuc}$ for the IS of Moskalenko et al. (2002), but not below $\sim 300 \mathrm{MeV} /$ nuc, although the $\mathrm{B} / \mathrm{C}$ values as a function of energy were fitted. A more reasonable fit is obtained in this energy range by using the second set of IS of Moskalenko et al. (2003), but not between $\sim 0.5 \mathrm{GeV} /$ nuc and $\sim 2 \mathrm{GeV} /$ nuc for both B and C. The new IS seems somewhat too high above $\sim 1 \mathrm{GeV} /$ nuc but still too low below $\sim 1 \mathrm{GeV} /$ nuc. The peak between $1-2 \mathrm{GeV} / \mathrm{nuc}$ in the ratio of the two IS should rather occur around $400 \mathrm{MeV} /$ nuc. It is argued that, in order to fit the $50-200 \mathrm{MeV} /$ nuc and $400-2000 \mathrm{MeV} /$ nuc observations simultaneously, very peculiar heliospheric diffusion coefficients are needed that will not fit other cosmic ray spectra. Drift models produce clearly different spectra for consecutive solar minimum conditions but the effect seems not large enough to account for the mentioned differences. All factors taken into account, our modelling indicates that the IS for $\mathrm{B}$ and $\mathrm{C}$ need further refinement below $\sim 1 \mathrm{GeV} /$ nuc (to make them true local IS) in the sense that the contribution of a local interstellar component, as Moskalenko et al. (2003) proposed, is present but not yet refined enough. To explain the $\mathrm{B}$ and $\mathrm{C}$ observations at Earth over the entire observed energy range, this proposed additional component should cause the local IS to bend significantly to higher values below $\sim 1 \mathrm{GeV} /$ nuc. Voyager data may then assist to determine to what low energy values this contribution may contribute, because at Earth this kind of information is "wiped out" by the adiabatic energy losses.

These results illustrate that this numerical model with a TS can reasonably reproduce the modulation of cosmic ray boron and carbon between the outer boundary (heliopause) and Earth, and for both magnetic polarity cycles from solar minimum to moderate solar activity, similar to what was done before for protons, anti-protons, electrons, positrons and helium.

Acknowledgements. We thank I. Moskalenko for useful discussions and for making the interstellar spectra and data compilation for B and $\mathrm{C}$ available to us. We thank the SA National Research Founda- tion (GUN number 2053475) for partial financial support.

Topical Editor R. Forsyth thanks H. Fichtner and R. B. McKibben for their help in evaluating this paper.

\section{References}

Burger, R. A., Potgieter, M. S., and Heber, B.: Rigidity dependence of cosmic-ray proton latitudinal gradients measured by the Ulysses spacecraft: Implications for the diffusion tensor, J. Geophys. Res., 105, 27 447-27 456, 2000.

Caballero-Lopez, R. A. and Moraal, H.: Limitations of the force field equation to describe cosmic ray modulation, J. Geophys. Res., 109(A1), A01101:1-10, doi: 10.1029/2003JA010098, 2004.

Davis, A. J., Mewaldt, R. A., Binns, W. R., Christian, E. R., Cummings, A. C., George, J. S., Hink, P. L., Leske, R. A., von Rosenvinge, T. T., Wiedenbeck, M. E., and Yanasak, N. E.: On the Low Energy Decrease in Galactic Cosmic Ray Secondary/Primary Ratios, in AIP Conf. Proc., 528, Acceleration and Transport of Energetic Particles Observed in the Heliosphere, ed. R. A. Mewaldt, J. R. Jokipii, M. A. Lee, E. Möbius, and T. H. Zurbuchen, 421, 2000.

Engelmann, J. J., Ferrando, P., Soutoul, A., Goret, P., and Juliusson, E.: Charge composition and energy spectra of cosmic-ray nuclei for elements from Be to NI - Results from HEAO-3-C2, Astron. and Astrophys., 233, 96-111, 1990.

Ferreira, S. E. S. and Potgieter, M. S.: Galactic cosmic rays in the heliosphere, Adv. Space Res., 34(1), 115-125, 2004a.

Ferreira, S. E. S. and Potgieter, M. S.: Long-term cosmic-ray modulation in the heliosphere, Astrophys. J., 603, 744-752, $2004 \mathrm{~b}$.

Ferreira, S. E. S., Potgieter, M. S., Heber, B., and Fichtner, H.:Charge-sign dependent modulation over a 22-year cycle, Ann. Geophys., 21, 1359-1366, 2003.

Fichtner, H.: Anomalous Cosmic Rays: Messengers from the Outer Heliosphere, Space Sci. Rev., 95, 639-754, 2001.

Haasbroek, L. J., Potgieter, M. S., and le Roux, J. A.: Acceleration of galactic and Jovian electrons at the solar wind termination shock, Adv. Space Res., 19(6), 953-956, 1997.

Hattingh, M. and Burger, R. A.: A new simulated wavy neutral sheet drift model, Adv. Space Res., 16(9), 213-216, 1995.

Heber, B.: Modulation of galactic and anomalous cosmic rays in the inner heliosphere, Adv. Space Res., 27(3), 451-461, 2001.

Heber, B., Clem, J. M., Müller-Mellon, R., Kunow, H., Ferreira, S. E. S., and Potgieter, M. S.: Evolution of the galactic cosmic ray electron to proton ratio: Ulysses COSPIN/KET observations, Geophys. Res. Lett., 30, ULY6:110, doi:10.1029/2003GL017356, 2003.

Jokipii, J. R. and Kóta, J.: The polar heliospheric magnetic field, Geophys. Res. Lett., 16, 1-4, 1989.

Jokipii, J. R., Kóta, J., and Merényi, E.: The gradient of galactic cosmic rays at the solar wind termination shock, Astrophys. J., 405, 782-786, 1993.

Krimigis, S. M., Decker, R. B., Hill, M. E., Armstrong, T. P., Gloeckler, G., Hamilton, D. C., Lanzerotti, L. J., and Roelof, E. C.: Voyager 1 exited the solar wind at a distance of $\sim 85 \mathrm{AU}$ from the Sun, Nature, 426, 45-48, 2003.

Langner, U. W.: Effects of termination shock acceleration on cosmic rays in the heliosphere, Ph.D. Thesis, Potchefstroom University, South Africa, 2004.

Langner, U. W. and Potgieter, M. S.: Solar wind termination shock and heliosheath effects on the modulation of pro- 
tons and anti-protons, J. Geophys. Res., 109, A01103:1-12, doi:10.1029/2003JA010158, 2004a.

Langner, U. W. and Potgieter, M. S.: Effects of the solar wind termination shock and heliosheath on the heliospheric modulation of galactic and anomalous Helium, Annales Geophys., in press, 2004b.

Langner, U. W., Potgieter, M. S., and Webber, W. R.: Modulation of cosmic ray protons in the heliosheath, J. Geophys. Res., 108(A10), 8039: LIS 14-1, doi:10.1029/2003JA009934, 2003.

le Roux, J. A., Potgieter, M. S., and Ptuskin, V. S.: A transport model for the diffusive acceleration and modulation of anomalous cosmic rays in the heliosphere, J. Geophys. Res., 101, 47914804, 1996.

McDonald, F. B., Heikkila, B., Lal, N., and Stone, E. C.: The relative recovery of galactic and anomalous cosmic rays in the distant heliosphere: Evidence for modulation in the heliosheath, J. Geophys. Res., 105, 1-8, 2000.

McDonald, F. B., Stone, E. C., Cummings, A. C., Heikkila, B., Lal, N., and Webber, W. R.: Enhancement of energetic particles near the heliospheric termination shock, Nature, 426, 48-51, 2003.

Moskalenko, I. V., Strong, A. W., Ormes, J. F., and Potgieter, M. S.: Secondary antiprotons and propagation of cosmic rays in the galaxy and heliosphere, Astrophys. J., 565, 280-296, 2002.

Moskalenko, I. V., Strong, A. W., Mashnik, S. G., and Ormes, J. F.: Challenging cosmic-ray propagation with antiprotons: Evidence for a "fresh" nuclei component?, Astrophys. J., 586, 1050-1066, 2003.

Parker, E. N.: The passage of energetic charged particles through interplanetary space, Planetary Space Sci., 13, 9-49, 1965.

Potgieter, M. S. and Ferreira, S. E. S.: Effects of the solar wind termination shock on the modulation of Jovian and galactic electrons in the heliosphere, J. Geophys. Res., 107, SSH1: 1-9, 2002.

Potgieter, M. S. and Langner, U. W.: Heliospheric modulation of cosmic ray positrons and electrons: Effects of the heliosheath and the solar wind termination shock, Astrophys. J., 602, 9931001, 2004.

Potgieter, M. S., Burger, R. A., and Ferreira, S. E. S.: Modulation of cosmic rays in the heliosphere from solar minimum to maximum: A theoretical perspective, Space Sci. Rev., 97, 295-307, 2001.

Reinecke, J. P. L. and Potgieter, M. S.: An explanation for the difference in cosmic ray modulation at low and neutron monitor energies during consecutive solar minimum periods, J. Geophys. Res., 99, 14 761-14 767, 1994.
Scherer, K. and Fahr, H.J.: Solar cycle induced variations of the outer heliospheric structures, Geophys. Res. Lett., 30, 17:1-4, doi:10.1029/2002GL016073, 2003.

Steenkamp, R.: Shock acceleration as source of the anomalous component of cosmic rays in the heliosphere, $\mathrm{Ph} . \mathrm{D}$. thesis, Potchefstroom University, South Africa, 1995.

Stephens, S. A. and Streitmatter, R. E.: Cosmic-ray propagation in the galaxy: Techniques and the mean matter traversal, Astrophys. J., 505, 266-277, 1998.

Stone, E. C. and Cummings, A. C.: Estimate of the location of the solar wind termination shock, Proc. 27th Inter. Cosmic Ray Conf. (Hamburg, Germany), 10, 4263-4266, 2001.

Stone, E. C. and Cummings, A. C.: The approach of Voyager 1 to the termination shock, Proc. 28th Inter. Cosmic Ray Conf. (Tsukuba, Japan), 3889-3892, 2003.

Strong, A. W., Bennett, K., Bloemen, H., Diehl, R., Hermsen, W., Morris, D., Schoenfelder, V., Stacy, J. G., de Vries, C., Varendorff, M., Winkler, C., and Youssefi, G.: Diffuse continuum gamma rays from the galaxy observed by COMPTEL, Astron. Astrophys., 292, 82-91, 1994.

Strong, A. W., Moskalenko, I. W., and Reimer, O.: Diffuse continuum gamma rays from the galaxy, Astrophys. J., 537, 763-784, 2000.

Wandel, A., Eichler, D., Letaw, J. R., Silberberg, R., and Tsao, C. H.: Distributed reacceleration of cosmic rays, Astrophys. J., 316, 676-690, 1987.

Webber, W. R., Lockwood, J. A., McDonald, F. B., and Heikkila, B.: Using transient decreases of cosmic rays observed at Voyagers 1 and 2 to estimate the location of the heliospheric termination shock, J. Geophys. Res., 106, 253-260, 2001.

Webber, W. R., Lukasiak, A., and McDonald, F. B.: Voyager measurements of the charge and isotopic composition of cosmic ray $\mathrm{Li}, \mathrm{Be}$, and $\mathrm{B}$ nuclei and implications for their production in the galaxy, Astrophys. J., 568, 210-215, 2002.

Zank, G. P., and Müller, H. R.: The dynamical heliosphere, J. Geophys. Res., 108, SSH7: 1-15, doi:10.1029/2002JA009689, 2003.

Zhang, M.: Modulation of galactic cosmic rays at solar maximum: Observations, Adv. Space Res., 32, 603-614, 2003. 